\title{
Molecular Methods in Microbiology and their Clinical Application
}

\author{
Malhotra $\mathbf{S}^{1^{*}}$, Sharma $\mathbf{S}^{1}$, Bhatia NJK ${ }^{1}$, Kumar $\mathbf{P}^{2}$ and Hans $\mathbf{C}^{1}$ \\ ${ }^{1}$ Department of Microbiology, PGIMER and Dr RML Hospital New Delhi, India \\ ${ }^{2}$ CCRUM, New Delhi, India
}

*Corresponding author: Malhotra S, Department of Microbiology, PGIMER and Dr RML Hospital New Delhi, India, E-mail: drshalinimalhotra@yahoo.com

Received date: March 14, 2014, Accepted date: November 20, 2014, Published date: November 27, 2014

Copyright: ( 2014 Malhotra S, et al. This is an open-access article distributed under the terms of the Creative Commons Attribution License, which permits unrestricted use, distribution, and reproduction in any medium, provided the original author(s) and source are credited.

\begin{abstract}
Conventional microbiology is an inexpensive but protracted diagnostic method and there are certain limitations associated with it. Molecular methods have been increasingly incorporated in laboratories, particularly for the detection and characterization of isolates and for the diagnosis of diseases due to fastidious, slow growing, nonviable or non-cultivable organisms which cannot be detected by conventional culture techniques. The introduction of these techniques and their automation provides new opportunities for the clinical laboratory to affect patient care. These tests provide timely results which are useful for high-quality patient care at a reasonable cost and are associated with improvement in patients care. The use of amplification techniques such as polymerase chain reaction, real-time polymerase chain reaction or nucleic acid sequence-based amplification for micro-organisms detection, genotyping and quantification. Molecular methods are rapid and highly sensitive and specific. This review will focus on various molecular techniques which are performed in clinical microbiology laboratories and their clinical applications and therefore help in management of infectious diseases.
\end{abstract}

Keywords: Amplification techniques; Automation; Infectious diseases; Molecular methods

\section{Introduction}

Detection and identification of the causative infectious agent is a highly relevant issue in microbiological diagnostics. Conventional microbiology is an inexpensive but protracted diagnostic method and there are certain limitations associated with it. Molecular methods have been increasingly incorporated in laboratories, particularly for the detection and characterization of isolates and for the diagnosis of diseases due to fastidious, slow growing, non-viable or non-cultivable organisms which cannot be detected by conventional culture techniques [1]. Molecular methods are based on the basic chemistry of DNA replication. The advantages are rapid turn-around time and high sensitivity and specificity however they are expensive, will miss new organisms, and too sensitive to assess clinical significance and also must be matched by rigorous validation and quality control [2].

\section{Current applications of molecular techniques in microbiology}

Diagnosis of non-culturable agents: e.g. Human papilloma virus, Hepatitis B virus etc.

Fastidious, slow-growing agents: e.g. Mycobacterium tuberculosis, Legionella etc.

Highly infectious agents that are dangerous to culture: e.g. Francisella, Brucella, Coccidioidis immitis etc.

In situ detection of infectious agents: e.g. H. pylori, Toxoplasma gondii etc.

Organisms when present in small volume in the specimen: e.g. detection of HIV in antibody negative patients, intra-ocular fluid, forensic samples etc.
For monitoring of viral load (for disease prognosis and treatment response): e.g. cytomegalovirus, Hepatitis B and C virus, HIV etc.

Differentiation of antigenically similar agents: e.g. for detecting specific virus genotypes associated with human cancers ( Papilloma viruses)

Antiviral/ antibacterial drug susceptibility testing: e.g. HIV to assess drug resistance, Determination of resistant genes like Mec A gene, Van genes

For the purpose of molecular epidemiology: e.g. Identify point sources for hospital and community-based outbreaks and to predict virulence

For confirmation of culture

\section{Molecular Methods are Broadly Classified as}

Hybridisation methods- better for identification, not as sensitive as amplification methods

Amplification methods- improve the sensitivity due to amplification step.

Sequencing and enzymatic digestion of nucleic acids $[1,3,4]$.

\section{Nucleic acid hybridization methods}

Hybridization methods are based on the ability of two nucleic acid strands that have complementary base sequence (i.e. are homologous) to specifically bond with each other and form a double- stranded molecule (hybrid). Since the hybridization requires sequence homology, a positive hybridization reaction between two nucleic acid strands each derived from different source indicate genetic relatedness between the two organisms. Hybridization assays require that one nucleic acid strand is from the known organism while the other is derived from the organism to be identified or detected. The results of 
hybridisation are expressed as percent hybridization/ percent similarity/ percent relatedness $[4,5]$.

\section{Steps involved in hybridization reactions are}

\section{Production and labelling of single stranded probes}

Probes are labelled short nucleic acids with known nucleotide sequences designed to hybridize with the target nucleic acid. Labelling can be done using radioactive or non-radioactive labels. The common radioactive isotopes used for labelling include 32P, 35S, 125I. Even though radioactive isotope labels provide maximum sensitivity, their disadvantages include higher expense, difficulty in handling, health hazard, short shelf life and disposal issues. The non-radioactive labels include biotin, digoxygenin and acridinium ester. Each Biotin binds specifically to four molecules of avidin. Biotinylated probes are used to hybridize with the target and such probes are detected using avidin tagged enzymes. Addition of substrate results in production of coloured product, signaling the positive hybridization reaction. Other methods include use of fluorescein conjugated avidin or fluorescein conjugated antibody to biotin molecule. Hybridization is observed for fluorescence using UV light.

\section{Preparation of single stranded target nucleic acid}

The source of target nucleic acid can be microorganism from the clinical specimen or from the culture. The nucleic acid is extracted chemically or enzymatically. The nucleic acid is treated to stabilize as well as preserve structural integrity and then denatured (if DNA) to derive single strands.

\section{Mixture and hybridisation of target and probe nucleic acid}

The annealing of the target nucleic acid with the corresponding specific nucleotide probe under optimum conditions of temperature and salt concentration is called hybridization. RNA-RNA duplexes are more stable than RNA-DNA duplexes, which are more stable than DNA-DNA duplexes.

\section{Detection of hybridization reaction}

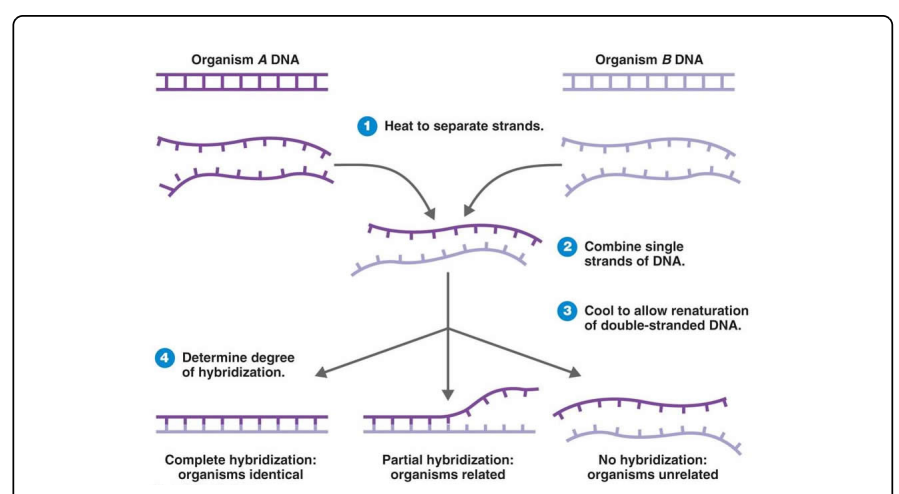

Figure 1: Digrammatic representation of hybridization technique for strain relatedness

The various methods of detection of hybridisation reaction are radiometric i.e. by autoradiography (radioactive isotope labelled probes), colorimetric (biotin or digoxygenin labelled probes), fluorometric (fluorescein tagged avidin or antibody) or chemiluminescence (acridinium ester labeled probe) as depicted in Figure 1.

\section{Hybridization is classified on the basis of its application into two types}

\author{
1. Solution format \\ 2. Solid Support format
}

\section{Solution phase hybridization}

The mixture of target nucleic acid and the probe are free to interact in an aqueous environment. The aqueous environment speeds up the rate of hybridization. The target DNA is denatured and the single stranded target nucleic acid is mixed with single stranded probes is added. Unhybridized single stranded nucleic acids are removed using S1 nuclease digestion. The hybridized dsDNA is recovered using trichloroacetic acid precipitation. Another approach is to bind the hybridized dsDNA to hydroxyapatite column, which binds to dsDNA selectively. Another common method called hybridization protection assay uses acridinium ester labelled probe to hybridize with the target. Upon addition of $\mathrm{H}_{2} \mathrm{O}_{2}$ hydroxide, the duplex emits light. This assay can be performed in few hours, because it does not require removal of excess of unbound single stranded DNA [4-6].

\section{Solid phase hybridization}

The hybridization reaction occurs on a solid support such as nitrocellulose or nylon membrane. The four known methods of hybridization on solid phase are dot/slot hybridization, sandwich hybridization, southern blot hybridization and in situ hybridization $[4,6]$.

\section{Dot hybridization}

Nucleic acid which is present in clinical samples in reasonable number (104-105) molecules/ml can be readily and specifically detected by dot hybridization. The target sample is affixed to a membrane in the form of dot or slot. The membrane is then processed to release target DNA from the micro-organism and denature it to a single strand. The membrane is immersed into a solution containing labelled probes and allowed to hybridize. Unbound probes are washed away and the hybridized duplexes are detected according to the nature of the reporter molecules. The advantages of this technique are that a single membrane can be used to test several specimens and a single specimen can be tested for several organisms on the same membrane.

\section{Sandwich hybridization}

It utilizes two probes; an unlabeled probe that is attached to a solid support (microtitre plate) and serves to capture the target from the sample to be tested. The presence of this duplex is then detected using a labelled second probe that is specific for another portion of the target sequence. Sandwiching the target between two probes decreases nonspecific reactions and increases specificity but is cumbersome due to greater number of processing and washing steps.

\section{Southern blot hybridization}

Once the DNA is extracted from the organisms and purified, it is cut into several fragments of various sizes using restriction enzymes. The resultant fragments are separated on gel by electrophoresis. The 
Page 3 of 9

fragments move along the gel according to their molecular weight, where the smaller fragments migrate faster and farthest through the gel than the larger fragments. The target DNA is transferred on to nylon or nitrocellulose membrane. The membrane is dipped in a hybridization fluid containing labeled probe. Deionized formamide, and detergents such as SDS are used to reduce non-specific binding of the probe. The hybridized duplexes are detected by radiometric, enzymatic or fluorometric methods depending on the nature of reporter molecule used. By this method, the bands can be seen on the membrane as depicted in Figure 2. This method allows the determination that which specific target nucleic acid fragment is carrying the base sequence of interest.

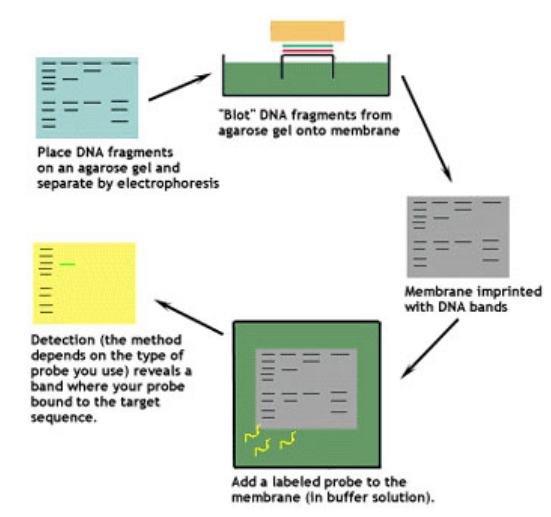

Figure 2: Diagram showing basic steps of southern blotting hybridization

\section{Northern blot hybridization}

This is similar to the southern blot in all aspects except that the target nucleic acid is single stranded RNA.

\section{In situ hybridization}

This allows a pathogen to be identified within the context of the pathologic lesion being produced. In in-situ hybridization the microscopy glass slide with tissue acts as the solid phase. Tissue specimens are processed in a way to maintain the structural integrity of the tissue and yet allow the nucleic acid of the pathogen to be released and denatured to a single strand with the base sequence intact. Radio-labeled probes and fluorescent probes (Fluorescence in situ hybridisation- FISH) are commonly used [4].

\section{Advantages of In situ hybridisation}

-Using this method it is possible to perform molecular hybridization while simultaneously viewing the histopathological structure of the specimen.

-This technique allows precise detection of infected cells in the tissue.

-This technique is useful in detecting intracellular parasites such as viruses and malignancies.

\section{Limitations of hybridisation techniques}

-Lacks sensitivity ( 104 copies of nucleic acid/ml for reliable detection) as compared to PCR/ amplification techniques $[1,4,5]$.

\section{Clinical applications of hybridization techniques}

Direct detection of pathogens e.g. in Pharyngitis (gp-A streptococci), genital tract infections (N.gonorrhoeae, C.trachomatis) by Gen Probe

\section{Speciation of microorganisms}

Identification of culture isolates e.g. Identification of dimorphic fungi, mycobacteria etc by AccuProbe.

\section{Amplification Techniques}

The beginning of molecular diagnostics was initiated at the end of the eighties with the development of the PCR [6]. Amplification methods improve the sensitivity due to amplification step. These are divided into three categories- target amplification, signal amplification and probe amplification $[7,8]$.

\section{Target amplification}

These systems amplify the target to large numbers. Some of these systems are:

\section{PCR - thermocycler is required}

Isothermal amplification methods: there is no need for thermocycler. Commonly used methods are:
NASBA (Nucleic acid sequence based amplification)
TMA (Transcription mediated amplification)
SDA (Strand displacement amplification)
LAMP (Loop mediated isothermal amplification)

\section{Polymerase Chain Reaction (PCR)}

PCR was invented by Kary Mullis in 1983 and received Nobel Prize in Chemistry for the same. PCR can enzymatically amplify minute quantities of DNA or RNA to large number of copies ( $\geq 107$ copies) in a short period hence provides ample target that can be readily detected. The basic steps in PCR are:

Denaturation of DNA to single strands $\left(94^{\circ} \mathrm{C}\right.$ or higher for 15 seconds to 2 minutes)

\section{Annealing of primers to DNA $\left(55^{\circ} \mathrm{C}, 1-2\right.$ mins)}

Extension by thermostable DNA polymerase $\left(72^{\circ} \mathrm{C}\right.$ typically $10-15$ minutes).

The same cycle is repeated for 30-35 times to produce approx. 109 copies / 2-3 hrs. All the steps of PCR are performed on the reaction mixture consisting of target DNA, primer pairs, thermostable DNA polymerase, deoxynucleotides (dATP, dTTP, dGTP and dCTP), buffer and $\mathrm{Mg}$ salt in the same test tube. The process of transferring the tubes to different temperatures has been circumvented by the use of automated machines called thermocyclers. After certain number of PCR cycles, PCR attains plateau phase. The plateau phase of PCR indicates that almost same amount of amplified products will be obtained, regardless of the initial amount of the templates, by sufficient cycles of PCR. Common problems in PCR are false negatives due to presence of PCR inhibitors, poor nucleic acid isolation and poor amplification efficiency and false positives due to contaminations. There are various methods to validate the PCR generated amplicons, as they serve to differentiate the target 
amplification from non-specific amplification [6,7]. These methods include:

- Electrophoresis of the amplicon should be sufficient in most cases to demonstrate similarity

between the expected and observed size of amplicons.

- Hybridization with probes for the known sequence within the amplified target sequence

- Nucleotide sequencing of amplicon

\section{Amplicon containment}

In order to limit the spread of amplicon from one PCR to another, multiple room approach is adopted. Preparation of primers, PCR buffers and dNTPs are done in room 1. Preparations of PCR assay except addition of target nucleic acid are done in room 2. Processing of specimen, preparation of target nucleic acid and addition of target to PCR assay are performed in room 3. PCR cycles for amplification are performed in room 4 and agarose gel electrophoresis of the amplicon is performed in room 5. The amplified products should never be carried to preceding rooms to avoid contamination $[7,8]$.

\section{Types of PCR}

RT-PCR (Reverse transcriptase- PCR): Since PCR can amplify dsDNA target sequences, single stranded RNA molecules cannot be amplified in the same way. In order to detect viral nucleic acid (of RNA viruses) in clinical specimen as well as to prepare cDNA library of mRNA, an additional step is performed before the PCR cycles are initiated. Using the enzyme reverse transcriptase, a complimentary copy of the RNA is made. A primer is annealed to the template RNA and with the help of reverse transcriptase, cDNA strand is synthesised by adding complementary base pairs. The template RNA is removed using the enzyme RNAse $\mathrm{H}$ leaving behind the newly generated single stranded cDNA, which can be amplified in PCR. A recombinant DNA polymerase derived from Thermus thermophilus (Tth pol) has both polymerase as well as RT activity in the presence of $\mathrm{Mn}^{2+}$ [9]. Commercially kits are available for detection of HCV RNA and for quantitation of HIV-1 and HCV RNA in clinical specimens (Roche Diagnostics, Indianapolis, IND).

Nested PCR: This modality of PCR increases both the sensitivity and specificity [10]. Nested PCR uses two sets of amplification primers. The first set of primers is used to amplify a target sequence and the second set of primers is used to amplify a region within the first target sequence. Essentially, this involves amplification of a sequence internal to an amplicon. Because the production of second amplicon depends on the successful production of the first amplicon, production of second amplicon automatically validates the accuracy of the first amplicon. Nested PCR may be performed in a single tube method or two- tubes method. Nested PCR makes the reaction very specific and alleviates false positive reactions that may occur with other PCR systems. The major disadvantage of nested PCR is the high rates of contamination [7].

Multiplex PCR: In multiplex PCR, two or more unique target sequences can be amplified simultaneously. It can be used in diagnostic assays that use one set of primers to amplify an internal control to verify the integrity of the PCR while the second set of primer is targeted to DNA sequence of interest. Absence of control amplicon indicates that PCR conditions were not met and the PCR may have to be repeated. Multiplex PCR can also be used to test for different organism on a single specimen. The primers are so designed that each amplification product is a unique size allowing detection and identification of specific organisms. Primer sets should have similar annealing temperatures. A limitation of multiplex PCR is that mixing different primers can cause some interference in the amplification process and the optimization of conditions becomes difficult as the number of primer pairs increase. Multiplex PCR assays for viral respiratory pathogens and for detection of viral infections of central nervous system have been developed and commercialized [11,12].

Competitive quantitative PCR (QPCR): Competitive PCR is a quantitative PCR, which is used to quantify the amount of nucleic acid in the sample. It involves a competition between the target nucleic acid and the competitive DNA for amplification process. The competitive DNA requires the same primer pair and is added in known concentration. It differs from the target DNA in size or presence of unique restriction site which is required to accurately differentiate target DNA from competitor DNA after the amplification process. Once amplification of target DNA is performed along with DNA competitor, competitive PCR occurs due to the competition for the use of the primers. So, the amount of the target DNA can be estimated by comparing with the concentration of DNA competitor $[1,4]$.

Real time PCR: The "real-time" PCR system discriminates from conventional PCR in following ways $[4,13,14]$ :

-They have the ability to detect amplified target DNA by fluorescently labeled probes as the hybrids are formed (i.e. detection of amplicon in real time). By plotting the increase in fluorescence versus cycle number, the system produces amplification plots that provide quantitative picture of the PCR process.

-Both amplification and product detection can be accomplished in one reaction vessel without ever opening hence cross contamination is lessened.

-Accumulation of amplicon is monitored as it is generated, hence able to quantitate the amount of product and also determine the number of copies of target in the original specimen.

Currently fluorescent probes are used for amplicon detection. SYBR Green I is non-specific and binds to ds DNA (both specific and nonspecific). Fluorescent probes used are Taqman probes, Molecular beacons and FRET (Fluorescent resonant energy transfer) hybridization probes $[4,15]$.

The probe is an oligonucleotide with both a reporter fluorescent dye at $5^{\prime}$ end and a quencher dye at $3^{\prime}$ end attached. As long as the probe is intact, the proximity of the quencher greatly reduces the fluorescence emitted by the reporter dye. If the target sequence is present, the probe anneals downstream from one of the primer sites and is cleaved by the 5 ' nuclease activity of Taq DNA polymerase as this primer is extended. This cleavage of the probe separates the reporter dye from quencher dye, increasing the reporter dye signal. More reporter dye molecules are cleaved from their respective probes with each cycle, leading to an increase in fluorescence intensity proportional to the amount of amplicon produced $[4,15]$.

The advantage of fluorogenic probes is that specific hybridization between probe and target is required to generate fluorescent signal. Thus nonspecific amplification due to mis-priming or primer-dimer artifact does not generate signal. Also fluorogenic probes can be labeled with different, distinguishable reporter dyes, hence amplification of two distinct sequences can be detected in a single PCR 
Page 5 of 9

reaction. The disadvantage of fluorogenic probes is that different probes must be synthesized to detect different sequences [4].

An amplification plot is the plot of fluorescence signal versus cycle number. In the initial cycles of PCR, there is little change in fluorescence signal which defines the baseline for the amplification plot. An increase in fluorescence above the baseline indicates the detection of accumulated PCR product. CT (threshold cycle) is defined as the fractional cycle number at which the fluorescence passes the fixed threshold and is dependent on the amount of target at the beginning of the reaction. Thus to quantitate the target in clinical specimen, a standard curve is generated in which known amounts of target are prepared and then subjected to real-time PCR along with the clinical sample containing an unknown amount of target [4].

\section{Clinical applications of Real-time PCR are}

-used to monitor the response to therapy

-to detect the development of drug resistance e.g. MRSA, VRE and

-to predict disease progression.

Helicase-dependent amplification: This technique is similar to traditional PCR, but uses a constant temperature rather than cycling through denaturation and annealing/extension cycles. DNA Helicase, an enzyme that unwinds DNA, is used in place of thermal denaturation.

\section{Transcription mediated amplification (TMA)/ Nucleic Acid Sequence Based amplification (NASBA)}

These are isothermal RNA amplification methods rather than DNA. Advantage of this technique includes performance of the test in isothermic conditions and no requirement of a thermocycler. The technique uses three enzymes in the reaction mixture; reverse transcriptase, RNase $\mathrm{H}$ and DNA dependent RNA polymerase. In TMA the enzyme used for reverse transcription also has intrinsic RNase $\mathrm{H}$ activity. So, in TMA only 2 enzymes used while in NASBA, 3 enzymes used. In this technique, the cDNA is created using reverse transcriptase and this is used as a template for transcription. A cDNA copy of the target RNA is made using a specific primer that binds to the target RNA. Reverse transcriptase builds a complementary DNA, resulting in the formation of RNA-cDNA hybrid. RNase enzyme digests and removes the RNA strand from the hybrid leaving behind single strand of cDNA. The second primer binds to the cDNA strand and the reverse transcriptase enzyme extends it using cDNA as template, resulting in generation of dsDNA copy of the target RNA. Using RNA polymerase, several copies of antisense RNA are produced from this dsDNA molecule. These RNA molecules can act as template for the reverse transcriptase resulting in the production of dsDNA molecules and the same cycle is repeated again $[3,16]$ (Table 1).

\begin{tabular}{|l|l|l|}
\hline S.No. & TMA & PCR \\
\hline 1. & Isothermal, no need of thermocycler & Require thermocycler \\
\hline 2. & Used for RNA amplification & For DNA amplification \\
\hline 3. & RNA is more labile so carryover contamination less & DNA is stable, hence more contamination and false positive results \\
\hline 4. & copies / cycle are produced & 2 copies / cycle are produced \\
\hline
\end{tabular}

Table 1: Showing difference between TMA and PCR

\section{Clinical applications of TMA/ NASBA}

To determine viral load in the sample e.g. HIV, Hepatitis B virus, Hepatitis $C$ virus.

\section{Monitor HCV patients on lamivudine}

Direct detection of M. tuberculosis in clinical samples.

Differentiate enteroviruses from other virus

\section{Strand Displacement Amplification (SDA)}

Strand displacement amplification is an isothermal DNA amplification reaction based on a restriction endonuclease nicking its recognition site and a polymerase extending the nick at its $3^{\prime}$ end, displacing the downstream strand. It requires restriction enzyme cleavage of the DNA sample prior to amplification in order to generate an amplifiable target fragment with defined 5 '- and 3 '-ends. Sample DNA is denatured at $95^{\circ} \mathrm{C}$ in the presence of excess of four specific primers that define target sequence. The primers S1 and S2 contain two regions, an unmodified HicII recognition site at their 5 ' ends that does not bind to the target and the other target specific region at its 3' end. S1 and S2 bind opposite strands of DNA flanking target sequence. The two other primers B1 and B2 are target specific, do not have any restriction site and bind to target upstream of primers S1 and S2. B1 and $\mathrm{B} 2$ primers are also extended and this result in the displacement of the strands extended from S1 and S2 primers. SDA products are more difficult than PCR products to decontaminate $[3,17]$ (Figure 3). 
Citation: Malhotra S, Sharma S, Bhatia NJK, Kumar P, Hans C (2014) Molecular Methods in Microbiology and their Clinical Application. J Mol

Target Generated containing engineered restriction enzyme site

\author{
Bumper Primer binds and displaces \\ strand generated by restriction \\ engineered primer
}

\section{Restriction Enzyme cleaves primer}

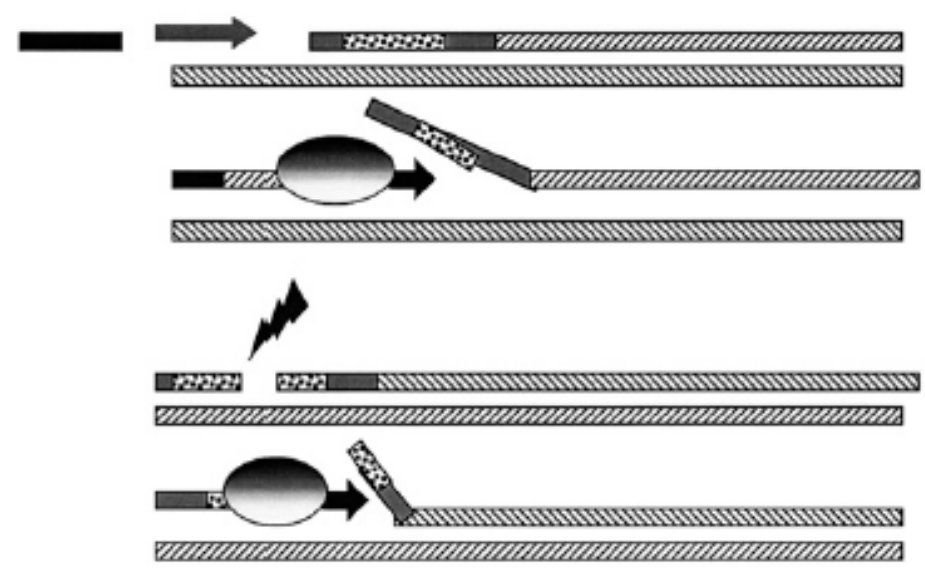

Figure 3: Showing steps of Strand displacement amplification

\section{LAMP (Loop Mediated Isothermal Amplification)}

This method relies on auto-cycling strand displacement DNA synthesis that is performed by a DNA polymerase and a set of two inner and two outer primers. In the initial steps, all four primers are used, but later during the cycling reaction only the inner primers are used for strand displacement DNA synthesis [18] (Table 2).

\begin{tabular}{|l|l|l|}
\hline S. No. & LAMP & PCR \\
\hline 1. & Simple isothermal test & Require thermocycler \\
\hline 2. & No need of electrophoresis, can be detected with unaided eye & Electrophoresis needed \\
\hline 3. & Duration -75 min & Duration $-4 \mathrm{hr}$ \\
\hline 4. & Rapid and cost effective method & costly \\
\hline
\end{tabular}

Table 2: Showing the difference between LAMP and PCR

\section{Clinical applications of LAMP}

bla 1 gene for ESBL detection

Detection of toxins-VT-E.coli, heat labile and heat stable toxin

OMP N gene of V.cholerae

Detection of C.jejuni and C. coli

Diagnosis of malaria (18S rRNA)

\section{Signal Amplification}

They amplify the signal generated by the labeled probes. By these procedures, a minimum 103-105 nucleic acid targets can be detected.

\section{Branched DNA (bDNA) probe system}

The key to assay is amplifier molecule with identical branches each of which can bind to three labelled probe i.e. capture probe, capture extendor (unlabelled) probe and labelled probe. The required nucleic acid target sequence is captured and hybridized with an unlabeled probe that has two hybridization sequences, one directed against the target sequence and the other capable of hybridizing with bDNA amplification multimer. This system is highly sensitive because the target nucleic acid has to bind both to the capture as well as target probes before the signals are amplified. Amplified signal is directly related to the number of targets present in the original sample [19]. 


\section{Nucleic Acid Capture Assay (NACA)}

Here the target molecule is directly captured onto the solid support and no branched oligodeoxyribonucleotides are used for detection and hence found more sensitive than bDNA assays [20]. NACA for detection of human papillomavirus (HPV) and cytomegalovirus $(\mathrm{CMV})$ in clinical specimens are currently commercially available (Digene Corp., Gaithersburg, MD).

\section{Probe amplification technique}

Probe amplification methods amplify products containing only the probe sequence. It includes Ligase Chain Reaction, Cycling Probe technology etc.

\section{Ligase Chain Reaction (LCR)}

LCR amplification is based on sequential rounds of template dependent ligation of two juxtaposed oligonucleotide probes. LCR allows the discrimination of DNA sequences differing in only a single base pair. Single stranded target DNA is incubated with oligonucleotide probes that bind to the target in an end-to-end fashion. A thermostable DNA ligase then ligates (or joins) the two probes together. The resulting duplex is heated to separate the target DNA and the ligated probes. Both the separated target sequence and the ligated probes now act as targets for the probes, which bind in an end-to-end fashion. These steps are repeated several times resulting in geometric probe amplification.

\section{Clinical applications of Ligase chain reaction}

For detection of certain bacteria like Borrelia burgdorferi, Listeria monocytogenes, Mycobacterium tuberculosis, Neisseria gonorrhoeae and Chlamydia trachomatis.

For isolation and identification of viruses e.g. Human papilloma virus, Herpes simplex virus, HIV etc.

${ }^{*}$ A combination LCR kit for detection of both Chlamydia trachomatis and Neisseria gonorrhea is now commercially available from Abbott Labs [21].

\section{Cycling Probe Technology (CPT)}

It employs a DNA-RNA-DNA fluorescent labelled probe at a constant temperature. The probe labelled with fluorescence one end and a Quencher the other, anneals to target DNA. An enzyme cuts the RNA region of the probe; the probe is no longer intact unquenching the signal, resulting in emission of fluorescence. Probe amplification is linear and not exponential. This application has been used to detect the mecA gene of MRSA [1].

\section{Sequencing and Enzymatic Digestion of Nucleic Acids}

DNA sequencing means determining the order of nucleotides in a DNA molecule for which extraction of plasmid or chromosomal DNA is done. This technique can be used to study the structure of gene, detect mutations, and compare genetic relatedness and to design oligonucleotide primers. Different techniques are given below:

\section{Restriction fragment length polymorphism}

Polymorphism (or variability) in nucleotide sequence is present in all organism including microbes. RFLP technique relies on the base pair changes in restriction sites, which arise due to mutations. Restriction enzymes (RE) cut DNA at specific 4-6 bp recognition sites and fragments separated according to molecular size by gel electrophoresis Ethidium bromide staining is used to reveal the fragments under UV $(260 \mathrm{~nm})$ light. Differences resulting from base substitutions, additions, deletions or sequence rearrangements within $\mathrm{RE}$ recognition sequences can be detected with this technique as depicted in Figure 4 [4] e.g. it can be used for determination of strain variation in M. tuberculosis [4,22].

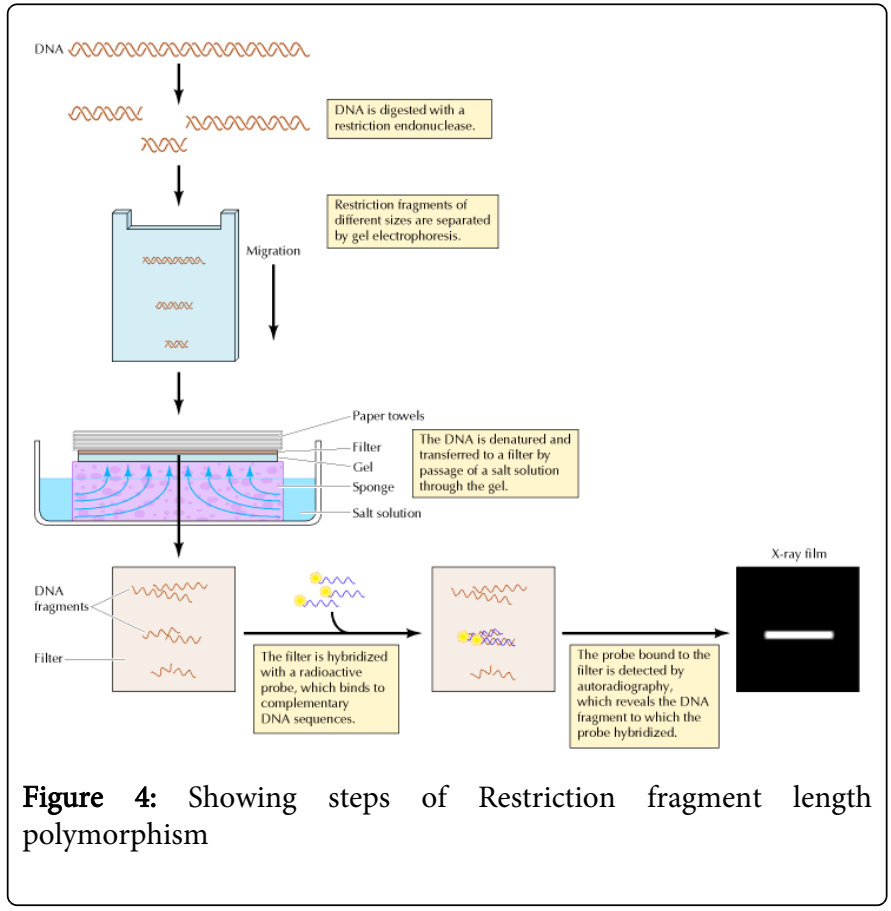

\section{Pulsed Field Gel Electrophoresis (PFGE)}

In PFGE, electrical fields are applied alternatively from different angles which lead to separation of large DNA fragments; hence there is enhanced resolution of fragments that differ by few bases.

\section{Other Molecular Methods}

\section{Line Probe Assay (LIPA)}

In LIPA, series of probes of interest are bound on nitrocellulose membrane/strip and amplicon is then applied to the strip which is just the reverse of southern blotting. Biotin labelled PCR product is then hybridised to the immobilised probes. After hybridisation streptavidin labelled with alkaline phosphatase is added and binds to the biotinylated hybrids.

\section{Clinical applications of LIPA}

$\mathrm{HCV}$ and HBV genotyping

Mutation associated with HIV

Mutation associated with mycobacteria

Detect HPV subtypes

Medically important fungi [23] 


\section{Ribotyping}

In ribotyping, restriction enzymes are used to cut the genes coding for rRNA into pieces, and then separated by gel electrophoresis. Universal probes that target specific conserved domains of ribosomal RNA coding sequences are used to detect the band patterns.

Clinical application: Allow subtype differentiation of bacterial isolates beyond the species and subspecies levels [3].

\section{Spoligotyping (spacer oligonucleotide typing)}

This technique is used as typing method for $M$. tuberculosis. Direct Repeats i.e. repeated sequences of base pairs are interspersed by nonrepetitive DNA spacers and these spacers are usually unique in a genome. Hence spacers are amplified and detected. It can be done on nonviable strains, stored samples or directly on samples [24].

\section{DNA Microarray}

It is a tool for analyzing gene expression. A DNA microarray (gene chip, DNA chip, or gene array) is a collection of microscopic DNA spots attached to a solid surface, such as glass, plastic or silicon chip (Probes). Each spot is designed to represent one gene and these are hybridised with cDNA or cRNA by forming hydrogen bonds between complementary nucleotide base pairs under high stringency conditions. A high number of complementary base pairs in a nucleotide sequence mean tighter non-covalent bonding between the two strands. Later it captures a snapshot of which genes are expessed in a given cell at a given time and monitors the whole genome on a single chip. Hence microarrays use relative quantitation in which the intensity of a feature is compared to the intensity of the same feature under a different condition [7].

\section{Clinical applications of microarray}

Genetic polymorphism of pathogen - distinguish among different strains of an isolate, detection of mutations in various isolates

Gene expression profile of pathogens: Pathogens adapt to different environmental conditions and different genes are expressed and some down regulated.

Identification of new targets for drug design and determination of their mechanism of action

Designing microarray for pathogen detection.

\section{Future of Molecular Diagnostic Techniques}

Despite being expensive, rapid diagnosis will result in decreased cost in the future.

Detection of Multidrug resistant M. tuberculosis will lead to more timely public health measures.

Increased specificity and sensitivity of molecular testing will become the standard of practice in immunology and microbiology

Testing will continue to become more rapid as assays are automated which will also bring down the costs.

\section{References}

1. Nolte FS, Caliendo AM (2003) Manual of Clinical Microbiology (8thedn). Murray et al.,(ed.), ASM Press, USA. Ch 17: 234-256.
2. Speers DJ (2006) Clinical applications of molecular biology for infectious diseases. Clin Biochem Rev 27: 39-51.

3. Tang YW, Procop GW, Persing DH (1997) Molecular diagnostics of infectious diseases. Clin Chem 43: 2021-2038.

4. Forbes BA, Sham DF, Weissfeld AS (2002) Nuceic acid based analytic methods for microbial identification and characterisation. Bailey and Scott's Diagnostic Microbiology, (12thedn).

5. Arnold LJ Jr, Hammond PW, Wiese WA, Nelson NC (1989) Assay formats involving acridinium-ester-labeled DNA probes. Clin Chem 35: 1588-1594.

6. Saiki RK, Gelfand DH, Stoffel S, Scharf SJ, Higuchi R, et al. (1988) Primer-directed enzymatic amplification of DNA with a thermostable DNA polymerase. Science 239: 487-491.

7. Cobo F (2012) Application of molecular diagnostic techniques for viral testing. Open Virol J 6: 104-114.

8. Van Leeuwen WB (2009) Molecular Diagnostics in Clinical Microbiology- Review article. Iran J Microbiol 1: 5-20.

9. Myers TW, Gelfand DH (1991) Reverse transcription and DNA amplification by a Thermus thermophilus DNA polymerase. Biochemistry 30: 7661-7666.

10. Haqqi TM, Sarkar G, David CS, Sommer SS (1988) Specific amplification with PCR of a refractory segment of genomic DNA. Nucleic Acids Res 16: 11844 .

11. Boriskin YS, Rice PS, Stabler RA, Hinds J, Al-Ghusein H, et al. (2004) DNA microarrays for virus detection in cases of central nervous system infection. J Clin Microbiol 42: 5811-5818.

12. Templeton KE, Scheltinga SA, Beersma MF, Kroes AC, Claas EC (2004) Rapid and sensitive method using multiplex real-time PCR for diagnosis of infections by influenza a and influenza B viruses, respiratory syncytial virus, and parainfluenza viruses $1,2,3$, and 4 . J Clin Microbiol 42: 1564-1569.

13. Morillo JM, Lau L, Sanz M, Herrera D, Silva A (2003) Quantitative realtime PCR based on single copy gene sequence for detection of Actinobacillus actinomycetemcomitans and Porphyromonas gingivalis. J Periodontal Res 38: 518-524.

14. Novais CM, Pires-Alves M, Silva FF (2004) PCR em tempo real. Rev Biotecnol Cienc Des ed. 33.

15. Valones MA, Guimarães RL, Brandão LA, de Souza PR, de Albuquerque Tavares Carvalho A, et al. (2009) Principles and applications of polymerase chain reaction in medical diagnostic fields: a review. Braz J Microbiol 40: 1-11.

16. Guatelli JC, Whitfield KM, Kwoh DY, Barringer KJ, Richman DD, et al. (1990) Isothermal, in vitro amplification of nucleic acids by a multienzyme reaction modeled after retroviral replication. Proc Natl Acad Sci U S A 87: 1874-1878.

17. Walker GT, Fraiser MS, Schram JL, Little MC, Nadeau JG, et al. (1992) Strand displacement amplification--an isothermal, in vitro DNA amplification technique. Nucleic Acids Res 20: 1691-1696.

18. Notomi T, Okayama H, Masubuchi H, Yonekawa T, Watanabe K, et al. (2000) Loop-mediated isothermal amplification of DNA. Nucleic Acids Res 28: E63.

19. Arens M (1993) Use of probes and amplification techniques for the diagnosis and prognosis of human immunodeficiency virus (HIV-1) infections. Diagn Microbiol Infect Dis 16: 165-172.

20. Mazzulli T, Drew LW, Yen-Lieberman B, Jekic-McMullen D, Kohn DJ, et al. (1999) Multicenter comparison of the digene hybrid capture CMV DNA assay (version 2.0), the pp65 antigenemia assay, and cell culture for detection of cytomegalovirus viremia. J Clin Microbiol 37: 958-963.

21. Chernesky MA, Lee H, Schachter J, Burczak JD, Stamm WE, et al. (1994) Diagnosis of Chlamydia trachomatis urethral infection in symptomatic and asymptomatic men by testing first-void urine in a ligase chain reaction assay. J Infect Dis 170: 1308-1311.

22. van Embden JD, Cave MD, Crawford JT, Dale JW, Eisenach KD, et al. (1993) Strain identification of Mycobacterium tuberculosis by DNA 
Citation: Malhotra S, Sharma S, Bhatia NJK, Kumar P, Hans C (2014) Molecular Methods in Microbiology and their Clinical Application. J Mol Genet Med 8: 142. doi:10.4172/1747-0862.1000142

Page 9 of 9

fingerprinting: recommendations for a standardized methodology. J Clin Microbiol 31: 406-409.

23. Johanna Makinen Marttila HJ, Marjamaki M, Viljanen MK, Soini $H$ (2006) Comparison of Two Commercially Available DNA Line Probe Assays for Detection of Multidrug-Resistant Mycobacterium tuberculosis. Journal of Clinical Microbiology 350-352.
24. Gori A, Bandera A, Marchetti G, Degli Esposti A, Catozzi L, et al. (2005) Spoligotyping and Mycobacterium tuberculosis. Emerg Infect Dis 11: 1242-1248. 